\title{
SPAG9 controls the cell motility, invasion and angiogenesis of human osteosarcoma cells
}

\author{
XIAORONG YANG ${ }^{1}$, WENLAI ZHOU ${ }^{2}$ and SHIQING LIU ${ }^{1}$ \\ ${ }^{1}$ Department of Orthopedics, Renmin Hospital of Wuhan University, Wuhan, Hubei 430060; \\ ${ }^{2}$ Department of Joints, Central Hospital of Tujia and Miao Autonomous Prefecture, Enshi, Hubei 445000, P.R. China
}

Received October 24, 2014; Accepted October 27, 2015

DOI: $10.3892 /$ etm.2015.2932

\begin{abstract}
Sperm-associated antigen 9 (SPAG9) is an oncoprotein involved in the progression of various human malignancies; however, its role in osteosarcoma (OS) remains poorly evaluated. The present study used Matrigel ${ }^{\mathrm{TM}}$ cell migration and invasion assays, tube formation assay, Cell Counting kit-8, quantitative polymerase chain reaction, western blotting, and enzyme-linked immunosorbent assay to investigate the role of SPAG9 in OS cell motility, invasion and angiogenesis. The results of the present study demonstrated that SPAG9 expression was upregulated in OS tissues, as compared with adjacent normal tissues, and knockdown of SPAG9 in an OS cell line inhibited cell motility and invasion via inactivation of metalloproteinase (MMP)-2 and MMP-9. Furthermore, the present study demonstrated that silencing of SPAG9 in OS cells inhibited tube formation, the proliferation of human umbilical vascular endothelial cells, and suppressed vascular endothelial growth factor (VEGF) expression and secretion, contributing to a reduction in angiogenesis. The results of the present study indicated that SPAG9 may be an important regulator in OS and may be involved in metastasis. Therefore SPAG9 may be a promising target for the treatment of metastatic OS.
\end{abstract}

\section{Introduction}

Osteosarcoma (OS) is a type of primary mesenchymal tumor, which is histologically characterized as a malignant tumor that directly produces osteoid or immature bones (1). Although OS remains the most common type of primary bone tumor in children and adolescents $(2,3)$, the 5-year survival rates of patients without metastases have reached $60-75 \%$

Correspondence to: Mr. Shiqing Liu, Department of Orthopedics, Renmin Hospital of Wuhan University, 238 Jiefang Road, Wuhan, Hubei 430060, P.R. China

E-mail: 13911391511@163.com

Key words: sperm-associated antigen 9, osteosarcoma, metastasis, metalloproteinase-2, metalloproteinase-9, vascular endothelial growth factor through the use of combined aggressive surgery and neoadjuvant chemotherapy (4). However, $40-50 \%$ of patients will develop metastatic disease, which is associated with a poor prognosis, particularly in patients with primary pulmonary metastases (5). Therefore, there is an urgent clinical need for novel therapies for the treatment of OS. Due to increased understanding regarding the molecular pathogenesis of OS, numerous genetic alterations have been associated with OS (6), which may represent novel therapeutic targets for the diagnosis and treatment of patients with OS.

Cancer testis (CT) antigens are a unique class of tumor antigens that are aberrantly expressed in various malignancies (7). It has been suggested that the aberrant expression of CT antigens in tumors may contribute to various malignant properties, including immortality, migration, invasion and metastatic capacity (8). As a novel member of the CT antigen family, sperm-associated antigen 9 (SPAG9) is a scaffolding protein that facilitates interactions between mitogen-activated protein kinases and their target transcription factors, in order to activate specific signaling pathways $(9,10)$. SPAG9 was first suggested as a potential target for immunotherapy in human epithelial ovarian cancer (11). Previous studies have detected overexpression of SPAG9 in various types of human cancer including renal, breast, thyroid, colon and lung carcinomas (12-16), and SPAG9 small interfering (si) RNA treatment successfully inhibited tumor cell proliferation and invasion in a previous study (12). Furthermore, SPAG9 expression has been demonstrated to be associated with circulating anti-SPAG9 antibodies in patients with early stage and low grade breast cancer and cervical cancer, suggesting its potential application in the early detection of the disease $(17,18)$. However, whether SPAG9 is involved in the development of OS remains to be elucidated. The present study aimed to determine the effects of SPAG9 knockdown on the proliferation, migration and invasiveness of OS cells in an in vitro cell culture system.

\section{Materials and methods}

Cell culture and tissue samples. The U2OS human OS cell line, and human umbilical vascular endothelial cells (HUVECs) were obtained from the Shanghai Academy of Life Sciences (Shanghai, China). The cells were cultured in Dulbecco's modified Eagle medium (DMEM) (HyClone; 
Thermo Fisher Scientific, Inc., Waltham, MA, USA) supplemented with $10 \%$ fetal bovine serum (FBS; Sigma-Aldrich, St. Louis, MO, USA) at $37^{\circ} \mathrm{C}$ in a humidified atmosphere containing $95 \%$ air and $5 \% \mathrm{CO}_{2}$.

Four OS tissue samples and paired non-cancerous tissue samples were harvested from patients with OS of the extremities (three men, one woman; age, 48-74 years) who underwent surgery at the Renmin Hospital of Wuhan University (Wuhan, China). None of the patients had a history of previous treatment with antitumor agents or radiotherapy. The patients provided informed consent and the study was approved by the ethics committee of Enshi Renmin Hospital of Wuhan University (Enshi, China).

siRNA transfection. U2OS cells were grown to 50\% confluence prior to transfection with nonspecific control siRNA (Qiagen, Inc., Mississauga, ON, Canada) or SPAG9 siRNA (Shanghai GenePharma Co., Ltd., Shanghai, China) using Lipofectamine ${ }^{\circledR} 2000$ transfection reagent (Invitrogen; Thermo Fisher Scientific, Inc.), according to the manufacturer's protocol. The siRNA sequences were as follows: Control, sense 5'-UUCUCCGAACGUGUCACGUTT-3', anti-sense 5'-ACGUGACACGUUCGGAGA ATT-3'; SPAG9, sense 5'-AGAUCUCAGUGGAUAUAAATT-3', and anti-sense 5'-UUUAUAUCCACUGAGAUCUTT-3'.

Wound healing assay. For the wound healing assay, $1 \times 10^{5}$ cells/well were seeded on 6 -well plates supplemented with culture medium. Cells were cultured in serum-free medium $12 \mathrm{~h}$ prior to the assay, and an artificial wound was subsequently scratched into the confluent cell monolayer using a P200 pipette tip. Photomicrograph images (TE2000; Nikon Corporation, Tokyo, Japan) were immediately captured (time $0 \mathrm{~h}$ ), and the cells were subsequently incubated in DMEM supplemented with $1 \%$ FBS. The migration of the cells and the closing of the scratch wound were observed and microphotographs were captured every 4 h (TE2000; Nikon Corporation). The experiments were performed in triplicate and the whole assay was repeated three times.

Cell migration and invasion assay. Modified two-chamber migration apparatus (pore size, $8 \mu \mathrm{m}$ ) was used to perform cell migration and invasion assays (Corning Life Sciences, Lowell, MA, USA). The migration assay was completed as follows, the U2OS cells transfected with control or SPAG9 siRNA were seeded in serum-free medium in the upper chamber $\left(1 \times 10^{6}\right.$ cells). Following $12 \mathrm{~h}$ incubation at $37^{\circ} \mathrm{C}$, the cells were carefully removed from the upper chamber using a cotton swab and the cells that had traversed the membrane were fixed. For the invasion assay, cells were trypsinized $48 \mathrm{~h}$ post-transfection and $2 \times 10^{5}$ cells were transferred in $100 \mu 1$ serum-free medium to the upper Matrigel ${ }^{\text {TM }}$ chamber (BD Biosciences, Franklin Lakes, NJ, USA) and incubated for $24 \mathrm{~h}$. Medium supplemented with 10\% FBS was added to the lower chamber as the chemoattractant. Following incubation, the non-invaded cells were removed from the upper membrane surface using a cotton tip, and the cells that passed through the filter were fixed with $4 \%$ paraformaldehyde and stained with hematoxylin (Sigma-Aldrich). Cells were observed under a light microscope (BX-51; Olympus
Corporation, Tokyo, Japan) and images were captured using a Camedia Master C-3040 digital camera (Olympus Corporation).

Cell proliferation assay. In order to quantitatively evaluate cell viability, the effects of si-SPAG9 on the proliferation of HUVECs and U2OS cells ( $2 \times 10^{4}$ cells/well) were detected using a CCK-8 assay. U2OS cells and HUVECs were seeded in a 96-well culture plate and incubated at $37^{\circ} \mathrm{C}$ in a humidified atmosphere containing $5 \% \mathrm{CO}_{2}$ for $24 \mathrm{~h}$. Subsequently, $10 \mu \mathrm{l}$ CCK-8 solution was added to each well and the cultures were incubated at $37^{\circ} \mathrm{C}$ for $1 \mathrm{~h}$. Absorbance was measured at $450 \mathrm{~nm}$ using an ELx800 spectrometer reader (BioTek Instruments, Inc.).

Tube formation assay. A 96-well plate was pre-coated with Matrigel ${ }^{\mathrm{TM}}$ and incubated at $37^{\circ} \mathrm{C}$ for $2 \mathrm{~h}$ prior to the addition of $2 \times 10^{4} \mathrm{HUVECs} /$ well suspended in $100 \mu \mathrm{l}$ conditioned medium. Following incubation at $37^{\circ} \mathrm{C}$ for $24 \mathrm{~h}$, three fields were chosen at random and the number of capillary-like tubes were counted. Images were captured using an inverted microscope (TE2000; Nikon Corporation).

Enzyme-linked immunosorbent assay (ELISA) for vascular endothelial growth factor (VEGF). A total of $1 \times 10^{6} \mathrm{U} 2 \mathrm{OS}$ cells/well were plated in 6-well tissue culture plates and subsequently transfected with control siRNA or siRNA-SPAG9 with serum starvation. The supernatants were collected $24 \mathrm{~h}$ post-transfection and the concentrations of VEGF were determined using Human VEGF Quantikine ELISA kits according to the manufacturer's protocol (R\&D Systems, Inc., Minneapolis, MN, USA).

Reverse transcription quantitative-polymerase chain reaction (RT-qPCR). Tissue samples were initially homogenized and total RNA was extracted from them using an mRNA Isolation kit (Qiagen, Inc., Valencia, CA, USA). An RNeasy kit (Qiagen, Inc., Valencia, CA, USA) was used to isolate mRNA from the cells. cDNA was synthesized from RNA extracted from cells and tissue samples using an iScript ${ }^{\mathrm{TM}}$ Select cDNA Synthesis kit (Bio-Rad Laboratories, Inc., Hercules, CA). PCR amplification of SPAG9, metalloproteinase (MMP)-2, MMP-9 and glyceraldehyde-3-phosphate dehydrogenase (GAPDH) was performed as follows: $20 \mathrm{sec}$ at $95^{\circ} \mathrm{C}$, followed by 50 cycles at $95^{\circ} \mathrm{C}$ for $3 \mathrm{sec}$ and annealing at $60^{\circ} \mathrm{C}$ for $30 \mathrm{sec}$, and a final extension step at $72^{\circ} \mathrm{C}$ for 5 min using $2 \mathrm{X}$ PCR Master Mix (Beyotime Institute of Biotechnology) and an ABI PRISM ${ }^{\circledR} 7500$ Sequence Detection system (Applied Biosystems; Thermo Fisher Scientific, Inc.). The results were normalized to those of the GAPDH housekeeping gene and are expressed as a ratio of the percentage of individual genes to the GAPDH control. The specific primer sequences for each gene were as follows: SPAG9 forward, 5'-TCATAG TAGAATTATTCAAGAG-3'; and reverse 5'-ATAGTAGAA TTATCTCTTGAA-3'; MMP-2 forward, 5'-CTGGCTTCT GGCATCCTGTT-3'; and reverse, 5'-GACGAGGTCGGA ATTGCAGA-3'; MMP-9 forward, 5'-CCTCTGGAGGTT CGACGTGA-3'; and reverse, 5'-TAGGCTTTCTCTCGG TACTGGAA-3'; and GAPDH forward, TGAAGGTCGGAG TCAACGGATT; and reverse, CCTGGAAGATGGTGATGG 
GATT (Shanghai GenePharma Co., Ltd.). Expression levels were analyzed using the $2^{-\Delta \Delta C q}$ method (19).

Zymographic analysis. Cells were grown to $70-80 \%$ confluence on $10 \mathrm{~cm}$ plates, washed twice with phosphate-buffered saline and cultured in serum-free medium for $36 \mathrm{~h}$. Total protein was extracted from the cells using radioimmunoprecipitation assay lysis buffer containing $60 \mu \mathrm{g} / \mathrm{ml}$ phenylmethylsulfonyl fluoiride (Beyotime Institute of Biotechnology). Protein concentrations were determined using a Bicinchoninic Acid Protein Assay kit (Boster Biological Technology, Ltd., Wuhan, China). An appropriate volume of medium with an equivalent amount of protein $(80 \mu \mathrm{g})$ was subjected to electrophoresis in $10 \%$ gel supplemented with $0.1 \%$ gelatin. Following electrophoresis, the gelatin gel was washed twice with $2.5 \%$ Triton $\mathrm{X}-100$ and incubated with Tris buffer $(50 \mathrm{mM}$ Tris- $\mathrm{HCl} ; 200 \mathrm{mM} \mathrm{NaCl}$; $0 \mathrm{mM} \mathrm{CaCl}$; pH 7.4) overnight at $37^{\circ} \mathrm{C}$. Subsequently, the gel was stained with $0.5 \%$ Coomassie Brilliant Blue R-250 and destained using 5\% acetic acid and 10\% methanol (all Invitrogen; Thermo Fisher Scientific, Inc.).

Western blot analysis. Tissue samples were homogenized, the homogenates were centrifuged at $1,000 \times \mathrm{g}$ for $10 \mathrm{~min}$ at $4^{\circ} \mathrm{C}$, and the supernatants were collected. Total protein was extracted from the cells and tissue supernatants using radioimmunoprecipitation assay lysis buffer supplemented with $60 \mu \mathrm{g} / \mathrm{ml}$ phenylmethylsulfonyl fluoride (Beyotime Institute of Biotechnology) $48 \mathrm{~h}$ post-transfection with siRNA. Protein concentrations were assessed using a Bicinchoninic Acid Protein Assay kit (Boster Biological Technology, Ltd.). Proteins $(80 \mu \mathrm{g})$ were separated by $10 \%$ sodium dodecyl sulfate-polyacrylamide gel electrophoresis, transferred to nitrocellulose membranes (EMD Millipore, Billerica, MA, USA) and incubated overnight at $4^{\circ} \mathrm{C}$ with the following antibodies: Rabbit anti-SPAG9 (1:1,000; cat. no. 5519), rabbit anti-MMP-2 (1:1,000; cat. no. 4022), rabbit anti-MMP-9 (1:1,000; cat. no. 3852) (all Cell Signaling Technology, Inc., Danvers, MA, USA), rabbit anti-VEGF (1:1,000; cat. no. sc-507) and mouse anti- $\beta$-actin (1:200; cat. no. sc-47778) (both Santa Cruz Biotechnology, Inc., Dallas, TX, USA). Blots were washed four times with Tris-buffered saline supplemented with $0.1 \%$ Tween-20 and subsequently incubated with horseradish peroxidase-conjugated anti-rabbit $(1: 2,000$; cat. no. 7074$)$ or anti-mouse (1:20,000; cat. no. 7076) secondary antibodies (Cell Signaling Technology, Inc.) at $24^{\circ} \mathrm{C}$ for $2 \mathrm{~h}$. The signals were detected using a Super Signal West Pico Chemiluminescent Substrate kit (Pierce Biotechnology, Inc., Rockford, IL, USA) according to the manufacturer's protocol. Western blots were analyzed using ImageJ $1.48 \mathrm{u}$ software (National Institutes of Health, Bethesda, MD, USA).

Statistical analysis. Data were expressed as the mean \pm standard deviation. Between group statistical differences were evaluated using Instat software (version 5.0; GraphPad Software, Inc., La Jolla, CA, USA) using one-way analysis of variance, whereas Student's t-test was used to analyze the cell proliferation assays. $\mathrm{P}<0.05$ was considered to indicate a statistically significant difference.

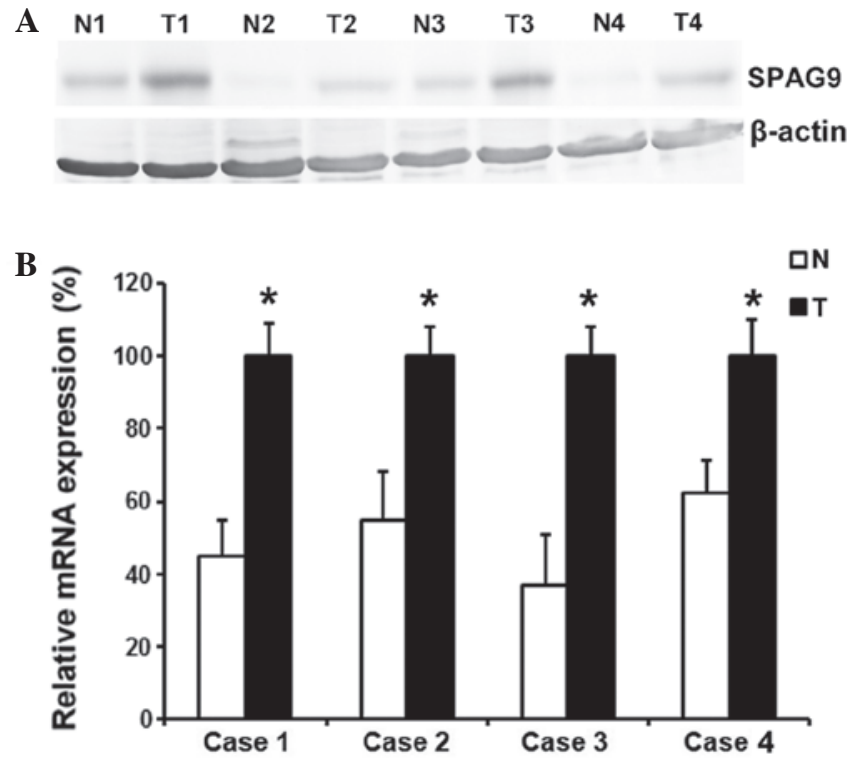

Figure 1. Sperm-associated antigen 9 (SPAG9) expression levels in normal (N) and cancerous osteosarcoma (T) tissues. (A) Whole-cell protein extracts were prepared from four pair-matched $\mathrm{N}$ and $\mathrm{T}$ tissues. SPAG9 protein expression levels were detected using western blot analysis. (B) SPAG9 mRNA expression levels in four pair-matched $\mathrm{N}$ and $\mathrm{T}$ tissues, as detected by reverse transcription quantitative-polymerase chain reaction analysis. Data are presented as the mean \pm standard deviation. ${ }^{*} \mathrm{P}<0.05$, as compared with $\mathrm{N}$.

\section{Results}

SPAG9 expression levels were upregulated in patients with osteosarcoma. In order to determine whether SPAG9 expression levels are altered in human patients with OS, RT-qPCR and western blotting were performed on four pairs of human OS tissue samples and pair-matched noncancerous tissue samples. The results demonstrated that SPAG9 expression levels were markedly increased in OS tissues, as compared with the noncancerous tissue samples (Fig. 1A and B).

Knockdown of SPAG9 by siRNA in U2OS cells inhibits cell motility. In order to explore the biological function of SPAG9 in OS cells, siRNA knockdown was performed in U2OS cell lines, and western blotting and RT-qPCR were used to analyze SPAG9 expression levels. Protein and mRNA expression levels of SPAG9 were markedly decreased $48 \mathrm{~h}$ following siRNA transfection (Fig. 2A and B). Therefore, all subsequent experiments were performed using cells transfected with SPAG9 or control siRNA.

The results of the cell migration assay demonstrated that transfection with SPAG9 siRNA decreased the migratory ability of U2OS cells through the Boyden chamber by $64 \%$ (Fig. 2C and D). Furthermore, the wound healing assay data demonstrated that there was a significant delay in wound closure following treatment with SPAG9 siRNA (Fig. 2E).

Knockdown of SPAG9 by siRNA in U2OS cells inhibits cell invasion. Invasion is the crucial step in the progression of cancer metastasis. The capacity of OS cells to invade through Matrigel $^{\mathrm{TM}}$, an artificial extracellular matrix (ECM), was measured following transfection with control or SPAG9 siRNA. Knockdown of SPAG9 expression resulted in a $48 \%$ 
A

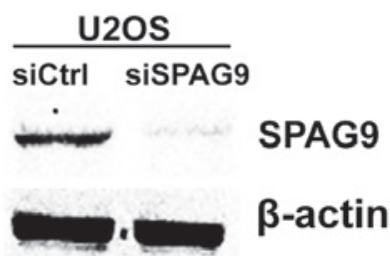

C

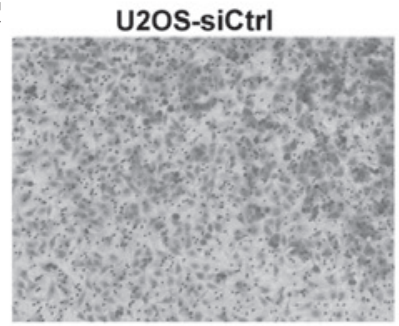

E
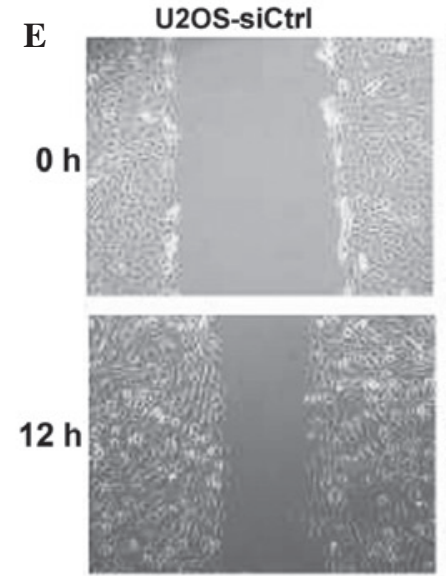

$24 \mathrm{~h}$

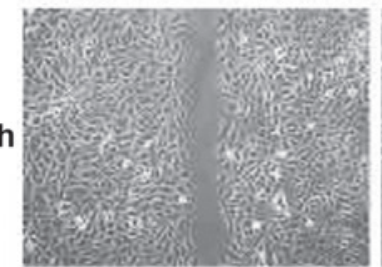

U2OS-siSPAG9

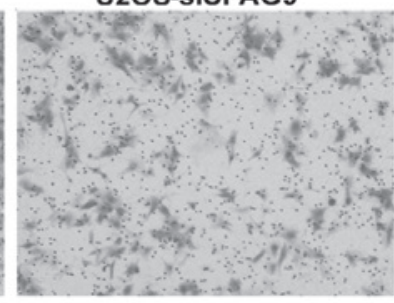

U2OS-siSPAG9
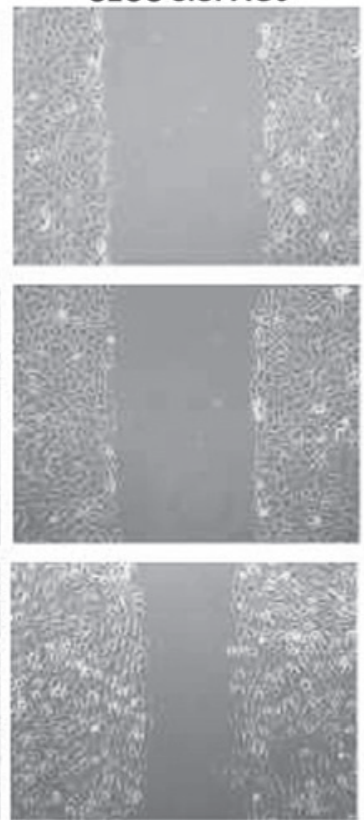

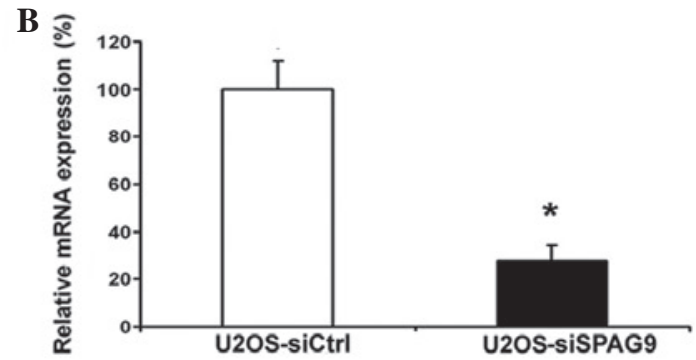

D
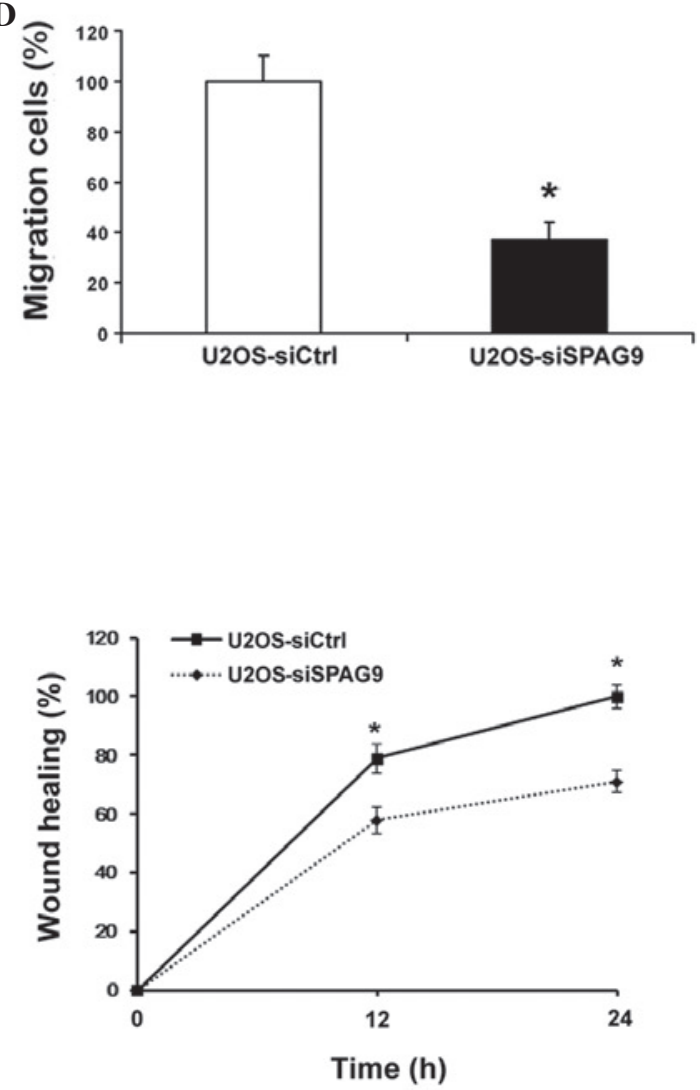

Figure 2. Sperm-associated antigen 9 (SPAG9) knockdown inhibited cell motility. (A) Protein expression levels of SPAG9 in U2OS cells were evaluated by western blotting $24 \mathrm{~h}$ post-transfection. $\beta$-actin was used as an internal control. (B) mRNA expression levels of SPAG9 in U2OS cells were examined by reverse transcription quantitative-polymerase chain reaction. Glyceraldehyde-3-phosphate dehydrogenase was used as an internal control. (C) Cell migration assay. Representative fields of migratory cells on the membrane (magnification, x200) and the (D) average number of migratory cells per field. (E) A scratch was made across the confluent monolayer using a $20 \mu 1$ pipette tip, and a subsequent wound healing assay demonstrated that there was a significant delay in wound closure following knockdown of SPAG9 expression (magnification, $\mathrm{x} 100$ ). Data are presented as the mean \pm standard deviation. ${ }^{*} \mathrm{P}<0.05$, as compared with the control (Ctrl). U2OS, human osteosarcoma cell line; si, small interfering RNA.

reduction in cell invasion (Fig. 3A), and histogram analysis demonstrated that significantly fewer cells $(\mathrm{P}<0.05)$ passed through the filters coated with an artificial ECM, suggesting that the invasive potential of SPAG9 siRNA-transfected OS cells was severely impaired. Downregulation of SPAG9 had no effect on the proliferation of OS cells (Fig. 3B).

Knockdown of SPAG9 inhibits angiogenesis in vitro. In order to further elucidate the functional role of SPAG9 on the angiogenic potential of human OS cells, tube formation and HUVEC proliferation were investigated in vitro. When cultured in conditioned medium from SPAG9 siRNA cells the percentage of proliferating HUVECs was significantly reduced (52\%; $\mathrm{P}<0.05$ ), as compared with the control (Fig. 4A). Furthermore, the average number of complete tubular structures formed by HUVECs cultured in conditioned medium from U2OS si-SPAG9 cells was significantly decreased $(\mathrm{P}<0.05)$, as compared with the control group (Fig. 4B).

Knockdown of SPAG9 suppresses MMP-2 and MMP-9 expression levels and activity in OS cells. MMPs, in particular MMP-2 and MMP-9, have important roles in the invasion and 
A
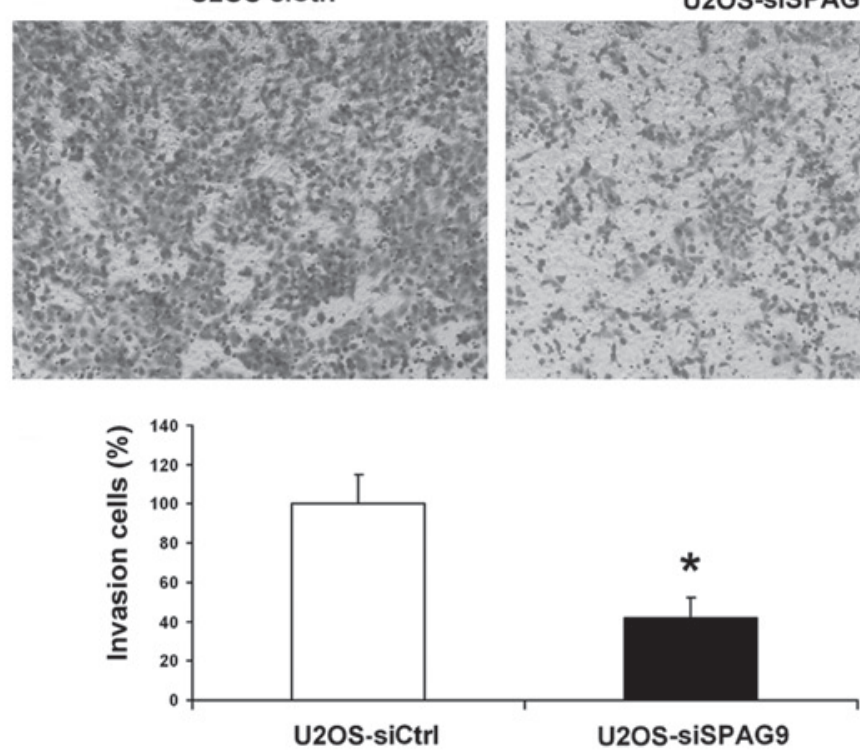

U2OS-siSPAG9

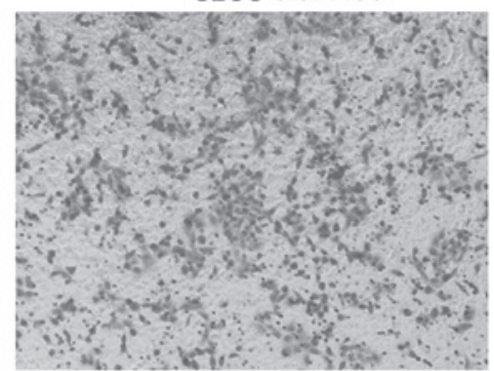

B

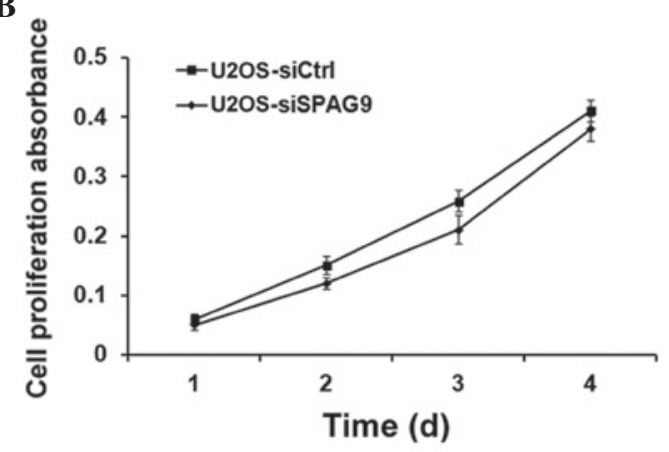

Figure 3. Sperm-associated antigen 9 (SPAG9) regulates cell invasion but not cell proliferation. (A) Matrigel ${ }^{\mathrm{TM}}$ cell invasion assay was performed following silencing of SPAG9 in U2OS cells (magnification, x100). (B) Cell Counting kit-8 cell proliferation assay was used to measure the proliferation of osteosarcoma (OS) cells. Data are presented as the mean \pm standard deviation in triplicate. "P $<0.05$, as compared with the control (Ctrl). si, small interfering RNA.

A

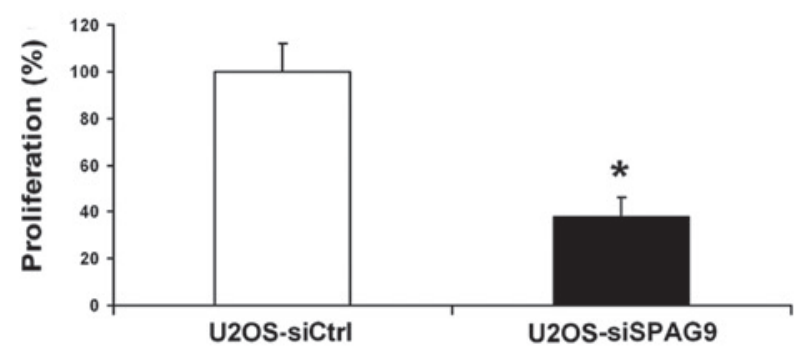

B
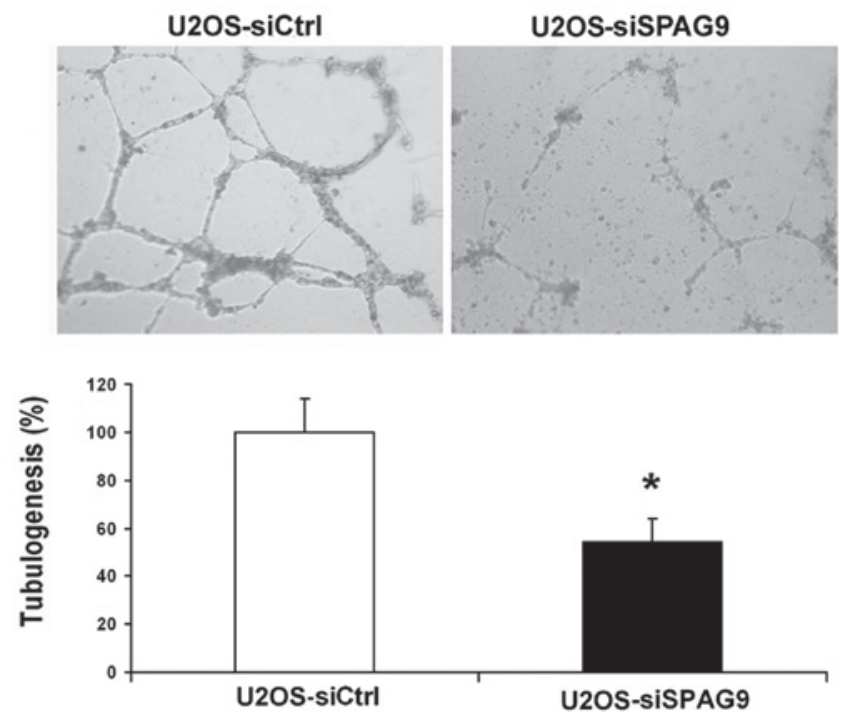

Figure 4. Sperm-associated antigen 9 (SPAG9) knockdown induced inhibition of angiogenesis. (A) Cell Counting kit-8 cell proliferation assay was performed to detect the proliferation of HUVECs following incubation with conditioned medium from SPAG9 small interfering (si)RNA U2OS cells. (B) Representative images were captured in situ of tube formation of HUVECs treated with the supernatant of U2OS cells transfected with control siRNA and si-SPAG9 (magnification, $\mathrm{x} 100)$. All experiments were performed in triplicate and data are presented as the mean \pm standard deviation ${ }^{*} \mathrm{P}<0.05$, as compared with the control (Ctrl). U2OS, human osteosarcoma cell line.

metastasis of OS cells. Therefore, RT-qPCR, western blot and gelatin zymography analyses were employed in order to determine the expression levels of MMP-2 and MMP-9 in si-SPAG9-transfected cancer cells. The results of the present study demonstrated that MMP-2 and MMP-9 mRNA and protein expression levels and activity were markedly reduced in U2OS cells transfected with si-SPAG9 (Fig. 5A-C), as compared with the control.

SPAG9 regulates VEGF expression and secretion in OS cells. VEGF is a key mitogen and survival factor in endothelial cells, as endothelial cells enter an active proliferative state in response to angiogenic stimulation. To evaluate whether depletion of SPAG9 contributes to VEGF expression and secretion in OS cells, a western blot analysis was performed in order to evaluate protein expression levels. A subsequent ELISA analysis was used to measure the secretion of VEGF in the conditioned medium from U2OS cells transfected with SPAG9 siRNA. A reduction in VEGF expression and secretion was detected in the conditioned medium from U2OS cells transfected with SPAG9 siRNA, as compared with the control cells (Fig. 6A and B). 
A

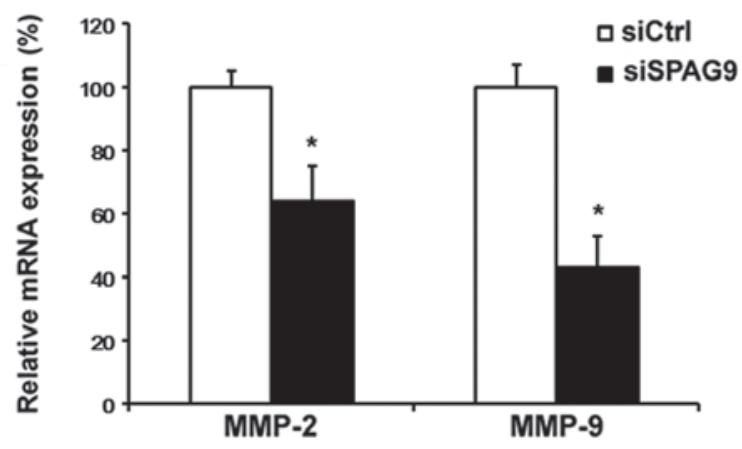

B

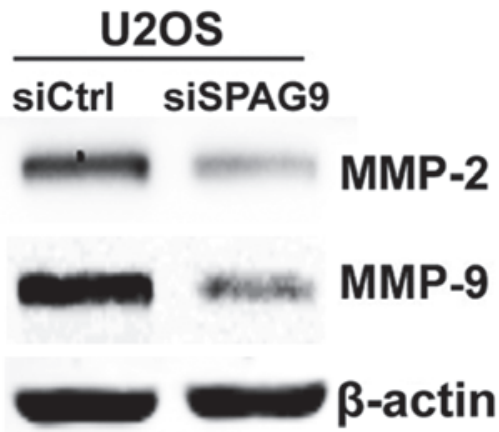

C

U2OS

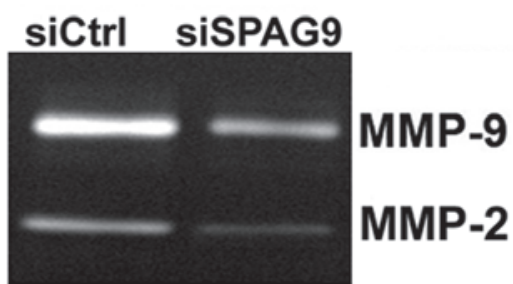

Figure 5. Sperm-associated antigen 9 (SPAG9) knockdown suppressed metalloproteinase (MMP)-2 and MMP-9 expression and activity levels. (A) Reverse transcription quantitative-polymerase chain reaction analysis of MMP-2 and MMP-9 mRNA expression levels following knockdown of SPAG9. (B) Western blot analysis of MMP-2, MMP-9 and $\beta$-actin relative protein expression levels in in the U2OS SPAG9 knockdown and control groups. (C) Activity levels of MMP-2 and MMP-9 were evaluated using gelatin zymography. Data are presented as the mean \pm standard deviation * $\mathrm{P}<0.05$, as compared with the control (Ctrl). U2OS, human osteosarcoma cell line; si, small interfering RNA.

\section{Discussion}

SPAG9 is localized on the surface of the sperm and is only expressed in haploid germ cells during spermatogenesis (9). Previous studies have demonstrated that SPAG9 may promote sperm-egg fusion, which is characterized by an increase in intracellular $\mathrm{Ca}^{2+}$ and $\mathrm{pH}$, and the tyrosine phosphorylation of several proteins $(20,21)$. Since SPAG9 is highly expressed in cancer tissues, the role of SPAG9 in cancer has been well-studied. Previous studies have suggested that SPAG9 may be useful as a tumor marker for various types of human cancer, including breast, thyroid, colorectal and renal cell carcinoma $(12,17,22)$. In addition, a previous study demonstrated that knockdown of SPAG9 inhibited tumor growth of renal cell carcinoma in vivo, indicating its potential role in the regulation of tumor development and metastasis (12). These observations indicated that SPAG9 may have an important role in the tumorigenesis of human cancer; however, the association between SPAG9 and OS has yet to be examined. In order to elucidate the role of SPAG9 in OS metastasis, tissue samples and an in vitro cell model were used to investigate whether SPAG9 is associated with the metastasis of OS. The results of the present study demonstrated that SPAG9 was highly expressed in OS tissues and knockdown of SPAG9 by siRNA in OS cells reduced cell migration, invasion and angiogenesis.

Metastasis is a complex multistep process that requires detachment from the primary tumor mass, migration through the ECM and the colonization of surrounding sites; therefore, cell motility and invasion are essential for this process (23). The present study demonstrated that knockdown of SPAG9 suppressed the motility and invasion of OS cells, as assessed
A
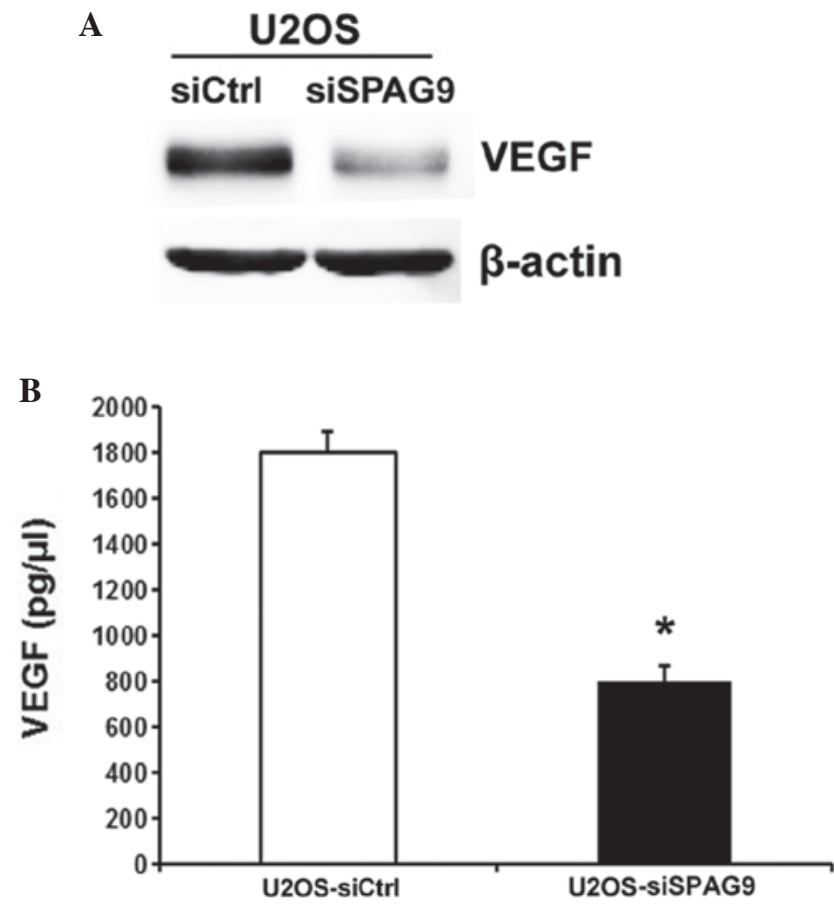

Figure 6. Sperm-associated antigen 9 (SPAG9) regulates vascular endothelial growth factor (VEGF) activity. (A) Western blot analysis of the relative protein expression levels of VEGF and $\beta$-actin in the U2OS SPAG9 knockdown and control groups. (B) VEGF secretion levels were evaluated using enzyme-linked immunosorbent assay. ${ }^{*} \mathrm{P}<0.05$, as compared with the control (Ctrl). U2OS, human osteosarcoma cell line; si, small interfering RNA.

by wound healing and transwell assays. One of the key factors associated with the invasion and metastasis of cancer cells is the degradation of ECM, as this allows cancer cells 
to invade and migrate. MMPs have an important role in this process (24), and MMP-2 and MMP-9 are postulated to promote the invasion and metastasis of OS cells to the lymph nodes (25). In order to determine how SPAG9 siRNA inhibits the motility and invasion of OS cells, the present study aimed to elucidate the effects of SPAG9 siRNA on MMP-2 and MMP-9 activity levels. Knockdown of SPAG9 expression significantly inhibited the expression levels and bioactivities of MMP-2 and MMP-9 in OS cells. The results of the present study indicated that SPAG9 may promote cell motility and invasion by increasing MMP-2 and MMP-9 expression levels and enzyme activity. However, it remains to be elucidated how SPAG9 regulates MMP-2 and MMP-9 expression and activity levels, and signaling pathway in order to regulate OS cell metastasis.

Angiogenesis is essential for the growth and metastasis of cancer cells. Previous studies have indicated that angiogenesis is associated with tumor metastasis in various types of cancer, including breast, lung, prostate, head, neck and melanoma (26-28). Therefore, the suppression of angiogenesis is emerging as a promising therapeutic strategy for the treatment of cancer (29). However, the effects of SPAG9 on angiogenesis are yet to be reported. The present study demonstrated that the transfection of SPAG9 siRNA reduced the capacity of OS cell supernatant to stimulate tube formation and proliferation of HUVECs, as compared with the control cells, suggesting that knockdown of SPAG9 expression levels significantly impaired the angiogenic potential of OS cells in vitro. However, the molecular bases for this hypothesis remain unclear. VEGF is considered a key mediator of tumor angiogenesis associated with the development of tumor blood supply and the progression of cancer (30). VEGF expression levels are downregulated by tumor suppressor genes, including p53, p75 and von Hippel-Lindau, which most likely occurs via the formation of complexes with Sp1, and the inhibition of its binding and subsequent transcriptional activation of the VEGF promoter $(31,32)$. The present study investigated whether SPAG9 regulated VEGF expression and activity levels in vitro. The results demonstrated that VEGF expression and secretion levels were decreased following SPAG9 knockdown, suggesting that decreased SPAG9 expression suppresses blood vessel formation by regulating VEGF expression and activity levels. Previous studies have demonstrated that SPAG9 participates in c-Jun N-terminal kinases (JNK) pathway activation, and JNK signaling may induce c-Jun phosphorylation at the VEGF promoter and result in its activation $(12,33)$; therefore it is possible that JNK signaling is involved in the angiogenesis-promoting function of SPAG9.

In conclusion, the key findings of the present study demonstrated that SPAG9 may be involved in promoting OS cell motility, invasion and angiogenesis. Silencing of SPAG9 led to the inhibition of motility and invasion in OS cells due to the inactivation of MMP-2 and MMP-9. Furthermore, knockdown of SPAG9 suppressed blood vessel formation and the proliferation of HUVECs, which may be associated with decreased secretion and expression of VEGF. These findings will not only advance our knowledge of OS biology, but may also help determine if SPAG9 has potential as a novel therapeutic target for the treatment of patients with OS.

\section{References}

1. Longhi A, Errani C, De Paolis M, Mercuri M and Bacci G: Primary bone osteosarcoma in the pediatric age: State of the art. Cancer Treat Rev 32: 423-436, 2006.

2. Nau JY: New techniques in bone and joint imaging (2). Rev Med Suisse 1: 651, 2005 (In French).

3. Klein MJ and Siegal GP: Osteosarcoma: Anatomic and histologic variants. Am J Clin Pathol 125: 555-581, 2006.

4. Hawkins DS and Arndt CA: Pattern of disease recurrence and prognostic factors in patients with osteosarcoma treated with contemporary chemotherapy. Cancer 98: 2447-2456, 2003.

5. Wu PK, Chen WM, Chen CF, Lee OK, Haung CK and Chen TH: Primary osteogenic sarcoma with pulmonary metastasis: Clinical results and prognostic factors in 91 patients. Jpn J Clin Oncol 39: 514-522, 2009.

6. Tan ML, Choong PF and Dass CR: Osteosarcoma: Conventional treatment vs. gene therapy. Cancer Biol Ther 8: 106-117, 2009.

7. Simpson AJ, Caballero OL, Jungbluth A, Chen YT and Old LJ: Cancer/testis antigens, gametogenesis and cancer. Nat Rev Cancer 5: 615-625, 2005.

8. Zendman AJ, Ruiter DJ and Van Muijen GN: Cancer/testis-associated genes: Identification, expression profile and putative function. J Cell Physiol 194: 272-288, 2003.

9. Jagadish N, Rana R, Selvi R, Mishra D, Garg M, Yadav S, Herr JC, Okumura K, Hasegawa A, Koyama K and Suri A: Characterization of a novel human sperm-associated antigen 9 (SPAG9) having structural homology with c-Jun N-terminal kinase-interacting protein. Biochem J 389: 73-82, 2005.

10. Jagadish N, Rana R, Mishra D, Kumar M and Suri A: Sperm associated antigen 9 (SPAG9): A new member of c-Jun NH2 -terminal kinase (JNK) interacting protein exclusively expressed in testis. Keio J Med 54: 66-71, 2005.

11. Garg M, Chaurasiya D, Rana R, Jagadish N, Kanojia D, Dudha N, Kamran N, Salhan S, Bhatnagar A, Suri S, et al: Sperm-associated antigen 9, a novel cancer testis antigen, is a potential target for immunotherapy in epithelial ovarian cancer. Clin Cancer Res 13: 1421-1428, 2007.

12. Garg M, Kanojia D, Khosla A, Dudha N, Sati S, Chaurasiya D, Jagadish N, Seth A, Kumar R, Gupta S, et al: Sperm-associated antigen 9 is associated with tumor growth, migration and invasion in renal cell carcinoma. Cancer Res 68: 8240-8248, 2008.

13. Sinha A, Agarwal S, Parashar D, Verma A, Saini S, Jagadish N, Ansari AS, Lohiya NK and Suri A: Down regulation of SPAG9 reduces growth and invasive potential of triple-negative breast cancer cells: Possible implications in targeted therapy. J Exp Clin Cancer Res 32: 69, 2013.

14. Volard B, Krieger S, Planchard G, Hardouin A, Vaur D, Rame JP and Bardet S: Assessment of SPAG9 transcript in fine needle aspirates of thyroid nodules. Eur Thyroid J 1: 118-121, 2012.

15. Kanojia D, Garg M, Gupta S, Gupta A and Suri A: Sperm-associated antigen 9 is a novel biomarker for colorectal cancer and is involved in tumor growth and tumorigenicity. Am J Pathol 178: 1009-1020, 2011.

16. Wang Y, Dong Q, Miao Y, Fu L, Lin X and Wang E: Clinical significance and biological roles of SPAG9 overexpression in non-small cell lung cancer. Lung Cancer 81: 266-272, 2013.

17. Kanojia D, Garg M, Gupta S, Gupta A and Suri A: Sperm-associated antigen 9, a novel biomarker for early detection of breast cancer. Cancer Epidemiol Biomarkers Prev 18: 630-639, 2009.

18. Garg M, Kanojia D, Suri S and Suri A: Small interfering RNA-mediated down-regulation of SPAG9 inhibits cervical tumor growth. Cancer 115: 5688-5699, 2009.

19. Livak KJ and Schmittgen TD: Analysis of relative gene expression data using real-time quantitative PCR and the 2(-Delta Delta C(T)) Method. Methods 25: 402-408, 2001

20. Burks DJ, Carballada R, Moore HD and Saling PM: Interaction of a tyrosine kinase from human sperm with the zona pellucida at fertilization. Science 269: 83-86, 1995.

21. Luconi M, Krausz C, Forti G and Baldi E: Extracellular calcium negatively modulates tyrosine phosphorylation and tyrosine kinase activity during capacitation of human spermatozoa. Biol Reprod 55: 207-216, 1996.

22. Garg M, Kanojia D, Salhan S, Suri S, Gupta A, Lohiya NK and Suri A: Sperm-associated antigen 9 is a biomarker for early cervical carcinoma. Cancer 115: 2671-2683, 2009. 
23. Hynes RO: Metastatic potential: Generic predisposition of the primary tumor or rare, metastatic variants-or both? Cell 113: 821-823, 2003

24. Halbersztadt A, Haloń A, Pajak J, Robaczynski J, Rabczynski J and St Gabryś M: The role of matrix metalloproteinases in tumor invasion and metastasis. Ginekol Pol 77: 63-71, 2006 (In Polish).

25. Zhang XY, Hong BF, Chen GF, Lu YL and Zhong M: Significance of MMP2 and MMP9 expression in prostate cancer. Zhonghua Nan Ke Xue 11: 359-361, 2005 (In Chinese).

26. Weidner N, Semple JP, Welch WR and Folkman J: Tumor angiogenesis and metastasis-correlation in invasive breast carcinoma. $\mathrm{N}$ Engl J Med 324: 1-8, 1991.

27. Weidner N: Intratumor microvessel density as a prognostic factor in cancer. Am J Pathol 147: 9-19, 1995.

28. Czubayko F, Schulte AM, Berchem GJ and Wellstein A: Melanoma angiogenesis and metastasis modulated by ribozyme targeting of the secreted growth factor pleiotrophin. Proc Natl Acad Sci USA 93: 14753-14758, 1996.
29. Folkman J: Tumor angiogenesis: Therapeutic implications. N Engl J Med 285: 1182-1186, 1971.

30. Cao Y, E G, Wang E, Pal K, Dutta SK, Bar-Sagi D and Mukhopadhyay D: VEGF exerts an angiogenesis-independent function in cancer cells to promote their malignant progression. Cancer Res 72: 3912-3918, 2012.

31. Xie K, Wei D, Shi Q and Huang S: Constitutive and inducible expression and regulation of vascular endothelial growth factor. Cytokine Growth Factor Rev 15: 297-324, 2004.

32. Pal S, Datta K and Mukhopadhyay D: Central role of p53 on regulation of vascular permeability factor/vascular endothelial growth factor (VPF/VEGF) expression in mammary carcinoma. Cancer Res 61: 6952-6957, 2001.

33. Guma M, Rius J, Duong-Polk KX, Haddad GG, Lindsey JD and Karin M: Genetic and pharmacological inhibition of JNK ameliorates hypoxia-induced retinopathy through interference with VEGF expression. Proc Natl Acad Sci USA 106: 8760-8765, 2009. 